\title{
ESTUDO PARAMÉTRICO DE SISTEMAS DE PISO BIDIRECIONAL E UNIDIRECIONAL MODULADOS EM CONCRETO ARMADO ${ }^{1}$
}

\author{
PARAMETRIC INVESTIGATION OF MODULAR BIDIRECIONAL AND \\ UNIDIRECIONAL REINFORCED CONCRETE FLOORS SYSTEMS
}

\author{
Bruno Leão de Brito \\ Universidade Federal da Bahia (UFBA) \\ leaodebrito@gmail.com \\ Felipe Tavares da Silva \\ Universidade Federal da Bahia (UFBA) \\ felipe.tavares@ufba.br
}

\begin{abstract}
Resumo
Com o crescente desenvolvimento das ferramentas digitais, sobretudo daquelas que implementam algoritmos gerativos, surge a necessidade de parametrizar os sistemas estruturais com o objetivo de verificar em quais situações estes podem ser melhor empregados. Os sistemas de piso em concreto armado formados por lajes sobre vigas são bastante utilizados na concepção de edificações, entretanto há pouca ou nenhuma informação sobre o consumo de material associado ao vão que cada tipologia demanda. Considerando alguns tipos de sistemas de piso modulado como protótipo de referência, foram investigados os índices de consumo de materiais e de impacto econômico e ambiental. Como resultado, foi criado um banco de dados com as taxas de consumo dos materiais necessárias para a construção de cada protótipo, relacionando-os com o vão.
\end{abstract}

Palavras-chave: Sistemas de piso. Concreto. Projeto paramétrico.Índices de desempenho.

\begin{abstract}
With the increasing development of digital tools, especially those that implement generative algorithms, the need to parameterize the structural systems in order to verify in which situations they can have better performance. Floor systems in reinforced concrete formed by slabs on beams are widely used in the design of buildings, however there is little or no information about the performance ratio of such floor systems. Considering some floor systems typologies modulated as a reference prototype, material consumption ratios and economic and environmental impact were investigated. As a result, a database was built with the consumption rates of materials necessary for the construction of each prototype, relating them to the span.
\end{abstract}

Keywords: Floor systems. Reinforced Concrete. Parametric design. Performances ratios.

\section{INTRODUÇÃO}

A sustentabilidade é um tema bastante presente em diversas áreas de estudos da atualidade, com enfoques ambientais, econômicos e sociais, e na área da AEC (Arquitetura, Engenharia e Construção) o cenário não é diferente. A existência de uma série de Selos e

\footnotetext{
1 BRITO, Bruno Leão de; SILVA, Felipe Tavares da. Estudo Paramétrico de Sistemas de Piso Bidirecional e Unidirecional Modulados em Concreto Armado. In: ENCONTRO BRASILEIRO DE TECNOLOGIA DE INFORMAÇÃO E COMUNICAÇÃO NA CONSTRUÇÃO, 7., 2015, Recife. Anais. Porto Alegre: ANTAC, 2015.
} 
controles de qualidade, como o LEED (Leadership in Energy and Environmental Design), e vários outros só mostram a necessidade de um melhor controle e uma melhor metodologia de trabalho na indústria da construção civil.

Rebelo (2007) apresenta ábacos de pré-dimensionamentos de lajes e vigas, separadamente, a partir de ábacos traduzidos de Corkill (1969). Entretanto estes ábacos não especificam quais são as resistências e os níveis de carga para cada ábaco como também não levam em consideração a interação das duas peças entre si. Isto pode levar a imprecisões no pré-dimensionamento, podendo acarretar modificações sucessivas desnecessariamente no processo de projeto.

Oliveira et al. (2013) obtiveram indicadores de consumo de materiais para edifícios em concreto armado entre 6 e 18 pavimentos, com lajes maciças em concreto armado e área de planta entre $260 \mathrm{~m}^{2}$ a $900 \mathrm{~m}^{2}$. Estes autores relataram que as taxas de consumo para sistemas de piso com lajes maciças e nervuradas apoiadas em vigas e do tipo cogumelo com vãos entre $4,0 \mathrm{~m}$ e $5,5 \mathrm{~m}$, geraram valores da taxa de concreto entre 0,15 e $0,21 \mathrm{~m}^{3} / \mathrm{m}^{2}$, e taxa de aço ente 8,49 e $19,89 \mathrm{Kg} / \mathrm{m}^{2}$.

Pereira et al. (2013), relataram índices de edificações entre 20 e 35 pavimentos em concreto armado, concebidos em lajes maciças e nervuradas, com áreas de planta entre $400 \mathrm{~m}^{2} \mathrm{e}$ $1900 \mathrm{~m}^{2}$ e vãos entre $3,0 \mathrm{~m}$ e 4,8 m. Estes obtiveram valores de taxa de concreto entre 0,11 e $0,19 \mathrm{~m}^{3} / \mathrm{m}^{2}$ e taxas de armadura entre 6,59 e $12,65 \mathrm{Kg} / \mathrm{m}^{2}$.

Tavares (2006) listou taxas de quantidades das estruturas de concreto generalizadas, relatando valores do consumo de concreto que variam de $0,05 \mathrm{~m}^{3} / \mathrm{m}^{2}$ para estruturas até 4 pavimentos a $0,22 \mathrm{~m}^{3} / \mathrm{m}^{2}$ para estruturas de 11 a 30 pavimentos. As taxas de aço relacionadas foram de $4,75 \mathrm{Kg} / \mathrm{m}^{2}$ a $28,6 \mathrm{Kg} / \mathrm{m}^{2}$ e $95 \mathrm{Kg} / \mathrm{m}^{3}$ a $130 \mathrm{Kg} / \mathrm{m}^{3}$, respectivamente.

Além do consumo de material, há na literatura alguns relatos de taxas de energia e $\mathrm{CO} 2$ incorporado ao concreto armado.Tavares (2006) lista valores de energia incorporada e emissão de $\mathrm{CO} 2$ relativos ao consumo de concreto e aço das armaduras. Foi relatado por este autor que para cada $\mathrm{m}^{3}$ de concreto são incorporados $2760 \mathrm{MJ}$ de energia enquanto que cada $\mathrm{Kg}$ de aço de armadura possui $30 \mathrm{MJ}$ de energia incorporada ao seu processo de fabricação do material. Além disto, ele relata que para cada $\mathrm{m}^{3}$ de concreto são emitidos 45 $\mathrm{Kg}$ de $\mathrm{CO} 2$, e para cada $\mathrm{Kg}$ de aço são emitidos $2,46 \mathrm{Kg}$ de $\mathrm{CO} 2$.

Por outro lado, a base de dados espanhola do ITEC (Instituto de Tecnología de La Construccíon) relata que para cada $\mathrm{m}^{3}$ de concreto são incorporados na sua produção 1462 MJ de energia e $271 \mathrm{Kg} \mathrm{CO}$, enquanto que para cada $\mathrm{Kg}$ de armadura são incorporados 37 $\mathrm{MJ}$ de energia e 3,00 $\mathrm{Kg}$ de $\mathrm{CO} 2$.

Adicionalmente aos índices de consumo, pode-se analisar as estruturas sob o índice de carbono e energia incorporados por metro quadrado de planta. Graf e Tavares (2010) obtiveram para uma edificação modelo, apenas pavimento térreo e de pequeno porte (184 $\mathrm{m}^{2}$ ), um valor de $1427 \mathrm{MJ}$ de energia incorporada por $\mathrm{m}^{2}$ da planta da estrutura. Oliveira et al. (2013) investigaram estruturas em concreto com número de pavimentos entre 20 a 35 , com áreas de pavimento entre $400 \mathrm{~m}^{2}$ e $900 \mathrm{~m}^{2}$, e relataram uma taxa de 1130 a $1700 \mathrm{MJ} / \mathrm{m}^{2}$ e uma taxa de 115,64 a 134,72 $\mathrm{Kg} \mathrm{CO} 2 / \mathrm{m}^{2}$ referentes aos casos investigados.

Neste artigo, tratamos de um experimento numérico que relaciona as opções de projeto com a energia incorporada no processo. Onde tem-se como primeira fase de desenvolvimento o pré-dimensionamento dos elementos do sistema de piso com base no método dos Estados Limites com o objetivo de gerar protótipos de unidades básicas pré-dimensionadas. $O$ método de pré-dimensionamento pelos Estados Limites se caracteriza como um processo de cálculo no sentido inverso ao apresentado pelas normas de dimensionamento de estruturas (SILVA,2013). Em seguida, os protótipos pré-dimensionados foram modelados, calculados e verificados no TQS, servindo tanto como validação da formulação proposta, 
como também de base para extração dos valores de consumo de materiais (aço, concreto e fôrmas). O software TQS é uma plataforma BIM (Building Information Modeling) desenvolvido para auxiliar e automatizar o dimensionamento e detalhamento de pórticos em concreto armado.

Foram investigados sistemas de piso com lajes bidirecionais e duas tipologias com lajes unidirecionais, considerando dois vãos de viga em cada direção para que se caracterizasse a continuidade das vigas. Em todos os casos de estudo, foram consideradas sobrecargas totais mínimas de $2,5 \mathrm{KN} / \mathrm{m}^{2}$ e máximas de $6,0 \mathrm{KN} / \mathrm{m}^{2}$. Estes dois níveis de sobrecarga vêm representar um nível de utilização residencial de $1,5 \mathrm{KN} / \mathrm{m}^{2}$ e uma carga mais elevada de $5,0 \mathrm{KN} / \mathrm{m}^{2}$ (NBR 6120,1980 ), adicionando em ambos os casos $1,0 \mathrm{KN} / \mathrm{m}^{2}$ de revestimento da laje. O nível mais elevado de carga de utilização poderia ser o caso de usos com estas características (clubes, bibliotecas, teatros, etc.) ou até mesmo a consideração de divisórias de alvenaria no meio da laje. As resistências características do material foram definidas de acordo com as classes de resistência recomendadas pela norma de dimensionamento de estruturas em concreto, a NBR-6118 (2003).

\section{PARAMETRIZAÇÃO DAS UNIDADES BÁSICAS DO SISTEMA DE PISO}

Vários autores (CORKILL,1969; MACDONALD,1998; ENGEL, 2001; ALLEN e IANO,2002; REBELLO,2007; CHING et. al.,2010) propõem método de pré-dimensionamento de estruturas a partir do método de determinação das espessuras estruturais através de ábacos, de percentuais em relação ao vão ou através de faixas de valores de vãos e espessuras estruturais. Em geral estes autores não relatam as origens destas relações entre espessura estrutural, com a exceção de Corkill (1969), dizendo que os seus ábacos foram gerados através de dados de edificações já construídas ou de soluções analíticas.

Ábacos de pré-dimensionamento são muito úteis para se verificar qual é a capacidade de vão de um determinado sistema estrutural, e sobretudo saber rapidamente qual é a espessura associada àquele vão. Contudo, os ábacos propostos pela literatura não especificam objetivamente qual o nível de cargas nem as resistências dos materiais. Outra limitação dos ábacos é que estes são geralmente de peças isoladas, gerando discrepâncias no caso de vigas, por exemplo, onde dependendo do tipo de laje que se apóia, esta irá receber um nível de sobrecarga distinto.

O processo de pré-dimensionamento de uma estrutura tem por objetivo gerar um conjunto que atenda aos requisitos dos Estados Limites de Serviço e Último. Com base nesta assertiva, pode-se afirmar que o atendimento dos requisitos dos Estados Limites significa verificar se os deslocamentos máximos estruturais são inferiores aos deslocamentos admissíveis $\left(\delta_{\max } \leq \delta_{a d m}\right)$ e se as solicitações de projeto são inferiores às resistências de projeto $\left(S_{d} \leq R_{d}\right)$.

Considerando as solicitações de projeto sendo obtidas a partir de soluções analíticas de esforços e deslocamentos máximos, limitadas às resistências de projeto fornecidas pela norma NBR6118 (2003), podem ser obtidas expressões de pré-dimensionamento dadas em função da carga, do vão, das dimensões das seções transversais e da elasticidade e resistência dos materiais.

Este trabalho tem por finalidade investigar três tipologias de sistemas de piso de lajes sobre vigas em concreto armado através de unidades básicas moduladas, como mostram as ilustrações da Figura 1. 
Figura 1 - Esquemas em planta das unidades básicas dos sistemas de piso investigados:

(a) Bidirecional; (b) Unidirecional 2M; (c) Unidirecional 3M.

(a)

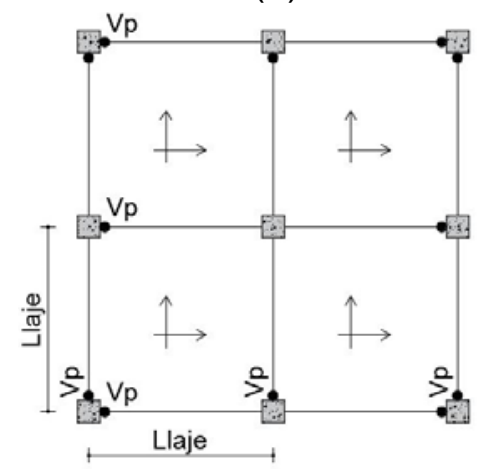

(b)

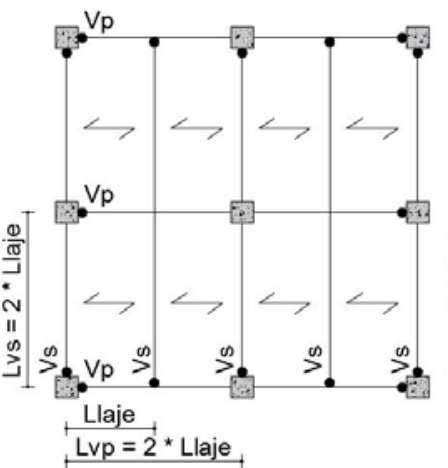

(c)

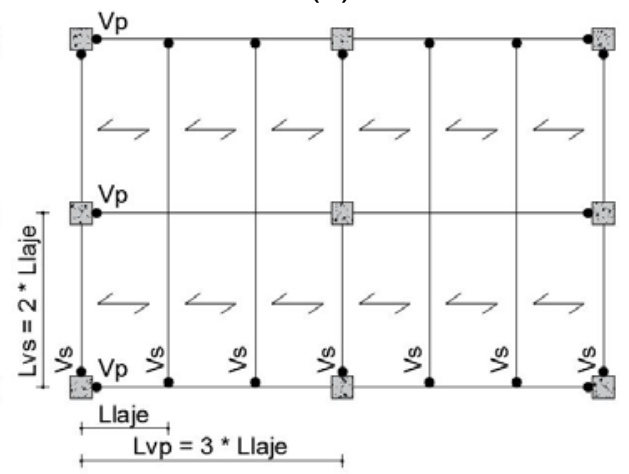

Fonte: elaborada pelos autores.

Estas unidades básicas de sistemas de piso são formadas por linhas de vigamentos ortogonais, considerando que os seus apoios extremos são rotulados, recebendo lajes bidirecionais ou unidirecionais. No caso das tipologias com lajes unidirecionais (Figura $1 \mathrm{~b}$ e c), foram consideradas vigas principais que apóiam uma viga secundária no meio do vão (módulo 2M) e um outro caso com duas vigas se apoiando no meio do vão (módulo 3M).

As tipologias de lajes bidirecionais consideradas foram a laje maciça, com espessuras entre $10 \mathrm{~cm}$ e $30 \mathrm{~cm}$; a laje nervurada treliçada com inter-eixo de $49 \mathrm{~cm}$, espessuras de $12 \mathrm{~cm}, 16$ $\mathrm{cm}, 20 \mathrm{~cm}, 25 \mathrm{~cm}$ e $30 \mathrm{~cm}$, com espessuras da capa de $4 \mathrm{~cm}$ ou $5 \mathrm{~cm}$; e lajes nervuradas com formas de cubetas plásticas, com inter-eixos de 61 e $80 \mathrm{~cm}$, espessuras de $21 \mathrm{~cm}, 23$ $\mathrm{cm}, 26 \mathrm{~cm}, 31 \mathrm{~cm}$ e $35 \mathrm{~cm}$, todas com espessura de capa de $5 \mathrm{~cm}$. As tipologias das lajes unidirecionais consideradas foram a laje maciça com espessuras de $10 \mathrm{~cm}$ e $15 \mathrm{~cm}$; e lajes treliçadas de inter-eixo de $43 \mathrm{~cm}$, espessuras totais de $12 \mathrm{~cm}$ e $16 \mathrm{~cm}$, com espessura de capa de $4 \mathrm{~cm}$.

A limitação destas espessuras das lajes, definindo assim um domínio, tomou como pressuposto a limitação do tamanho da viga no entorno de $100 \mathrm{~cm}$, considerando que todas as vigas possuem uma relação entre espessura vertical e espessura horizontal de 1:3 no máximo.

\subsection{Parametrização das lajes}

De acordo com o pré-dimensionamento segundo os requisitos dos Estados Limites, foram desenvolvidas expressões analíticas que fornecem os vãos máximos das lajes em função da sua espessura, da resistência do concreto e do nível de cargas considerado. Há uma expressão de vão máximo para cada esforço relevante de projeto e para o deslocamento.

As expressões dadas pelas equações de [1] a [5] fornecem os vãos máximos para lajes bidirecionais e unidirecionais segundo as condições do momento fletor de projeto, esforço cortante de projeto e deslocamento admissível, respectivamente. O vão adotado para a laje deverá ser o menor dentre todos estes vãos críticos, de modo que atenda a todas as condições.

$$
L_{M F}^{\text {maciça }} \leq K_{M F} \cdot d \cdot \sqrt{\frac{f_{c k}}{q_{L A J E}}}
$$


$L_{M F}^{\text {nervurada }} \leq K_{M F} \cdot d \cdot \sqrt{\frac{e_{\text {capa }} \cdot f_{c k}}{q_{L A J E}} \cdot\left(4,91 \cdot d-2,04 \cdot e_{\text {capa }}\right)}$

$L_{E C} \leq K_{E C} \cdot \frac{f_{c k}^{2 / 3} \cdot A_{c o n c}}{q_{L A J E} \cdot i e}$

$L_{D E S 1} \leq K_{D E S 1} \cdot d \cdot\left(\frac{\sqrt{f_{c k}}}{q_{P P}+q_{R E V}+0,3 \cdot q_{V A R}}\right)^{1 / 3}$

$L_{D E S 2} \leq K_{D E S 2} \cdot d \cdot\left(\frac{\sqrt{f_{c k}}}{q_{V A R}}\right)^{1 / 3}$

$d_{E Q}=\left(\frac{12 \cdot I_{T}}{i e}\right)^{1 / 3}$

O Quadro 1 lista os valores das constantes que diferenciam as expressões para as tipologias de lajes consideradas.

Quadro 1 - Valores das constantes para lajes uni e bidirecionais.

\begin{tabular}{|c|c|c|c|c|}
\hline \multirow{2}{*}{ Constante } & \multicolumn{2}{|c|}{ Laje bidirecional } & \multicolumn{2}{c|}{ Laje unidirecional } \\
\cline { 2 - 5 } & Maciça & Nervurada & Maciça & Nervurada \\
\hline $\mathrm{K}_{\mathrm{MF}}$ & 0,36 & 0,38 & 0,27 & 0,24 \\
\hline $\mathrm{K}_{\mathrm{EC}}$ & 0,84 & 084 & 0,43 & 0,43 \\
\hline $\mathrm{K}_{\mathrm{DES} 1}$ & 0,47 & 0,39 & 0,34 & 0,34 \\
\hline $\mathrm{K}_{\mathrm{DES} 2}$ & 0,57 & 0,48 & 0,41 & 0,41 \\
\hline
\end{tabular}

Fonte: elaborado pelos autores.

As expressões de [1] a [5] fornecem os vãos críticos para as lajes dados em metros, em função da espessura total da laje $d$, de sua capa e, e inter-eixo ie no caso de lajes nervuradas, dadas em centímetros; resistência característica do concreto $f_{c k}$ em MPa; da carga total $q_{\text {LAJE }}$ ou de suas parcelas de peso próprio $q_{P P}$, de revestimento $q_{R E V}$ ou de utilização $q_{V A R}$ dadas em $\mathrm{KN} / \mathrm{m}^{2}$; e da área bruta da seção transversal $A_{\text {conc }} \mathrm{em} \mathrm{cm}^{2}$.

Para o cálculo do vão crítico sob a condição do deslocamento (Eqs. [4] e [5]) nos casos das lajes nervuradas deve ser considerada a espessura equivalente, dada em função do momento de inércia $I_{T}$ da seção $T$ formada com uma mesa com largura igual ao inter-eixo ie entre as nervuras.

As condições de apoio da laje bidirecional considerada foi com duas bordas contíguas engastadas e duas outras rotuladas, obtendo assim as expressões analíticas para os esforços máximos e deslocamento na laje para este caso. No caso das lajes unidirecionais foi considerado o caso de um apoio engastado e o outro rotulado.

A condição do deslocamento foi dividida em duas pelo fato da separação entre a combinação quase permanente e da ação isolada das cargas variáveis na análise de deslocamentos (CARVALHO e PINHEIRO,2013). Nos dois casos foi considerada uma redução na inércia por um fator de 3,0 devido à consideração da sessão fissurada. No caso da carga permanente, foi considerada uma amplificação de 2,5 vezes do deslocamento 
obtido para a consideração da fluência do concreto.

O deslocamento admissível para a comparação com a componente do deslocamento máximo devido às cargas permanentes mais $30 \%$ da variável [4] foi de $L / 250$ e para a comparação com o deslocamento máximo devido às cargas variáveis [5] foi de L/350.

\subsection{Parametrização das vigas contínuas}

Uma vez que os vãos das vigas são definidos em função da definição do vão máximo da laje (Figura 1), a parametrização da viga consiste em definir qual é a sua altura mínima em função do vão definido pela laje, da resistência do concreto, da dimensão da base da seção transversal e do nível de sobrecarga que esta recebe. As expressões de [7] a [10] fornecem as alturas mínimas necessárias sobre as condições do momento fletor, esforço cortante e deslocamento, respectivamente. A altura mínima adotada deve ser a maior dentre as críticas de modo que atenda a todas as condições.

$$
\begin{aligned}
& h_{V_{-} M F} \geq \frac{7 \cdot b_{V} \cdot L_{V}{ }^{2}+1,6 \cdot \sqrt{19,14 \cdot b_{V}{ }^{2} \cdot L_{V}{ }^{4}+7 \cdot 10^{7} \cdot C_{M} \cdot b_{V} \cdot L_{V}{ }^{2} \cdot f_{c k} \cdot\left(n \cdot R_{L A J E}+q_{A L V}\right)}}{32000 \cdot C_{M} \cdot b_{V} \cdot f_{c k}} \\
& h_{V_{-} E C} \geq \frac{0,875 \cdot L_{V} \cdot\left(n \cdot R_{L A J E}+q_{A L V}\right)}{\left(0,019-0,000077 \cdot f_{c k}\right) \cdot f_{c k} \cdot b_{V}-0,0022 \cdot b_{V} \cdot L_{V}} \\
& h_{V_{-} D E S 1} \geq \sqrt[3]{a+\sqrt{a^{2}+b}}-\sqrt[3]{-a+\sqrt{a^{2}+b}} \\
& \rightarrow\left\{\begin{array}{l}
a=\frac{1277 \cdot L_{V}{ }^{3} \cdot\left(n \cdot \left(R_{\text {LAJE_QPERM } \left.\left._{-}+0,3 \cdot R_{\text {LAJE }_{\text {VAR }}}\right)+q_{\text {ALV }}\right)}\right.\right.}{b_{V} \cdot \sqrt{f_{c k}}} \\
b=\frac{-9,7 \cdot L_{V}{ }^{9}}{\sqrt{f_{c k}{ }^{3}}}
\end{array}\right. \\
& h_{V_{-} D E S 2} \geq 11,27 \cdot L_{V} \cdot\left(\frac{n \cdot R_{L_{A J E} V A R}}{b_{V} \cdot \sqrt{f_{c k}}}\right)^{1 / 3} \\
& C_{M}=\left\{\begin{array}{l}
0,0001946 \rightarrow f c k \leq 35 M P a \\
0,0001638 \rightarrow f c k>35 M P a
\end{array}\right.
\end{aligned}
$$

Nestas expressões acima $b_{V}$ e $h_{V}$ são respectivamente a base e a altura da viga dadas em centímetros; $L_{v}$ é o vão da viga em metros; $f_{c k}$ a resistência característica do concreto em $\mathrm{MPa}$; n o número de lajes que se apóia na viga; $R_{L A J E}$ a reação de apoio da laje que se apóia em $\mathrm{KN} / \mathrm{m} ; q_{A L V}$ a carga linear de parede que está sobre a viga em $\mathrm{KN} / \mathrm{m}$ e $C_{M}$ um coeficiente que depende do valor da resistência do concreto.

Foi considerado para a determinação dos esforços e deslocamentos máximos analíticos o modelo de viga rotulada em um apoio e engastada no outro.

O cálculo da reação de apoio da laje $\left(R_{L A J E}\right)$ para o caso bidirecional pode ser admitido como $25 \%$ do produto da carga total na laje pelo vão da mesma e no caso unidirecional $50 \%$, considerando que o vão da mesma é a menor dimensão do painel da laje. Nas expressões da determinação da altura mínima das vigas na condição do deslocamento (Eqs. [9] e [10]), semelhantemente ao que foi estabelecido para as lajes, deve-se considerar as componentes de carga permanentes e variáveis distintas nos dois casos. As reações $R_{\text {LAJE_PERM }}$ e $R_{\text {LAJE_VAR }}$ são respectivamente as parcelas das reações de apoio da laje em relação à combinação 
quase permanente e em relação à ação isolada das cargas variáveis sobre a laje.

No caso das lajes unidirecionais, na determinação da altura mínima das vigas principais foram utilizadas as expressões [12] e [13] para a quantificação das sobrecargas das mesmas, substituindo $q_{S C}$ por $R_{\text {LAJE }}$ nas expressões de [7] a [12].

$$
\begin{aligned}
& q_{S C_{-} 2 M}=1,60 \cdot \frac{\left(q_{P P}^{\mathrm{sec}}+2 \cdot R_{L A J E}^{\text {unidirecional }}\right) \cdot L_{V S}}{L_{V P}} \\
& q_{S C_{-} 3 M}=2,75 \cdot \frac{\left(q_{P P}^{\mathrm{sec}}+2 \cdot R_{L A J E}^{\text {unidirecional }}\right) \cdot L_{V S}}{L_{V P}}
\end{aligned}
$$

Nas expressões [12] e [13] $q_{s C_{-} 2 M}$ e $q_{S C_{-} 3 M}$ são as sobrecargas que o sistema de vigas secundárias e laje atua sobre a viga principal de um sistema de piso com lajes unidirecionais do tipo $2 \mathrm{M}$ e $3 \mathrm{M}$, respectivamente; $L_{v s}$ e $L_{v P}$ são as dimensões dos vãos da viga secundária e principal respectivamente.

\section{VALIDAÇÃO DA PARAMETRIZAÇÃO E QUANTIFICAÇÃO DOS MATERIAIS}

Dentro das faixas de valores de espessuras de laje, resistência do concreto e níveis de sobrecarga considerados neste estudo, foram geradas dezenas de unidades básicas dos sistemas de piso nas tipologias ilustradas pela figura 1.

Foi utilizado o sistema TQS para realizar o cálculo, dimensionamento e quantificação do concreto e das armaduras de todas estas unidades básicas geradas. Uma vez que o sistema TQS segue as recomendações da norma (NBR6118,2003), o uso deste também serviu como etapa de validação das formulações propostas. Além do quantitativo dos materiais, foram extraídas as reações das vigas sobre os pilares centrais das unidades básicas com a finalidade de obter qual é o peso próprio aproximado dos sistemas de piso nestas tipologias.

\section{RESULTADOS}

Após o processamento de todas as tipologias geradas e modeladas no software TQS, obtendo assim os respectivos quantitativos de materiais, foram construídos gráficos que mostram a distribuição destes dados obtidos.

Graficamente, foram obtidas as relações entre espessura estrutural e vão principal, fornecendo o pré-dimensionamento dos sistemas nestas tipologias moduladas em função do vão principal das unidades básicas ilustradas pela Figura 1.

As Figuras 2 e 3 ilustram a relação entre as espessuras de lajes e vigas em relação ao vão do sistema estrutural para cada tipo de laje investigada.

Observa-se que as respostas obtidas pelas expressões de pré-dimensionamento propostas mostram proporção entre vão e espessura do sistema de piso próximos às propostas de Rebello (2007). Contudo, no caso das vigas que suportam as lajes bidirecionais e as vigas principais do sistema de piso com lajes unidirecionais, foram obtidas alturas maiores em relação ao vão, em comparação com os resultados de Rebello (2007). 
Figura 2 - Ábacos das lajes bidirecionais e suas vigas nas tipologias estudadas.
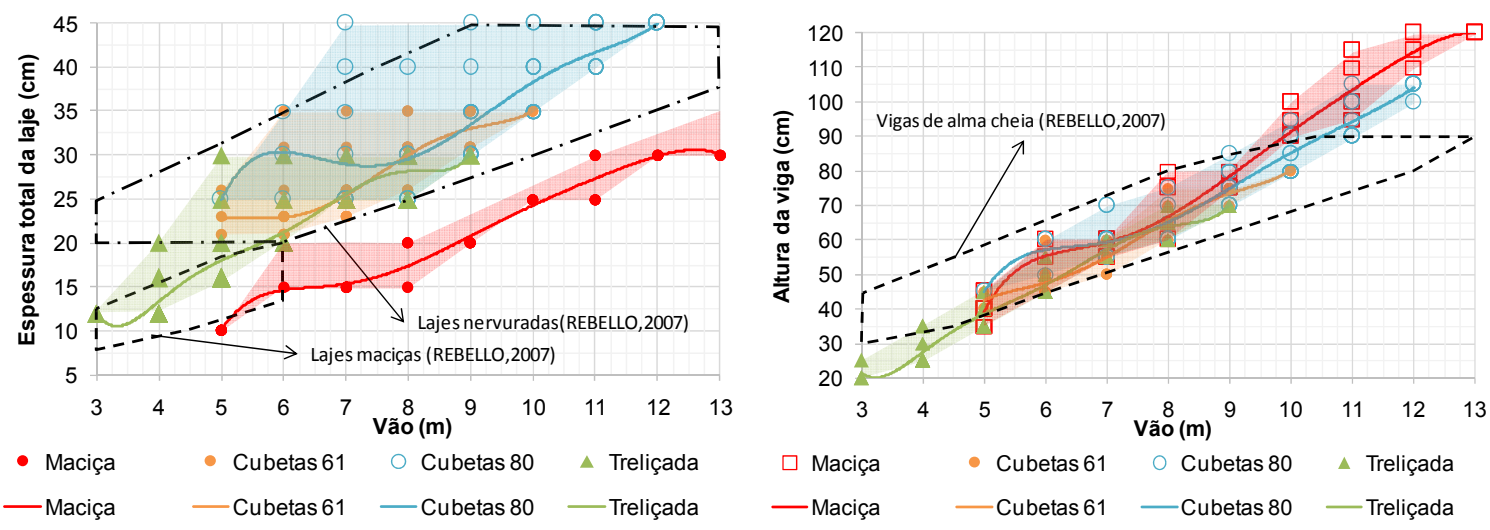

Fonte: elaborada pelos autores.

Figura 3 - Ábacos das lajes unidirecionais e suas vigas nas tipologias estudadas.
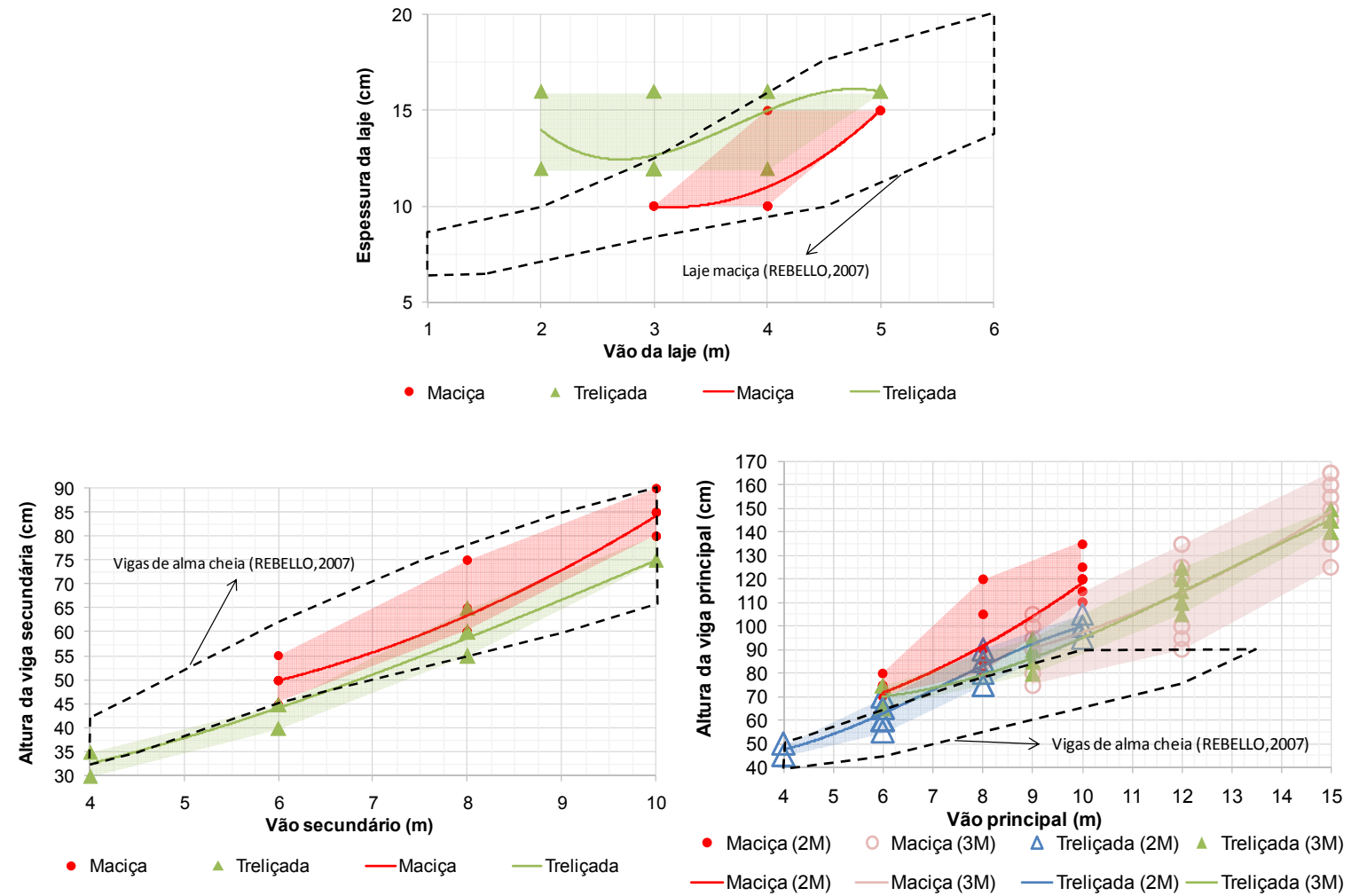

Fonte: elaborada pelos autores.

Em todas as tipologias estudadas, em todos os gráficos exibidos, foram traçadas linhas de regressão obtidas por polinômio de grau seis, sendo a escolha do grau atrelado ao maior valor do índice de correlação $\mathrm{R}^{2}$ associado. Estas linhas de tendência ilustram a variação média da espessura estrutural em função do vão.

Os índices de desempenho dos protótipos analisados variam de acordo com o vão principal do pórtico e estão ilustrados nas Figuras de 4 e 5, respectivamente para os sistemas de piso com laje bidirecional e unidirecional. Nestas figuras também estão marcadas as regiões com os valores de referência de outros estudos realizados por outros autores. 
Figura 4 - Índices de desempenho dos sistemas de piso com laje bidirecional.
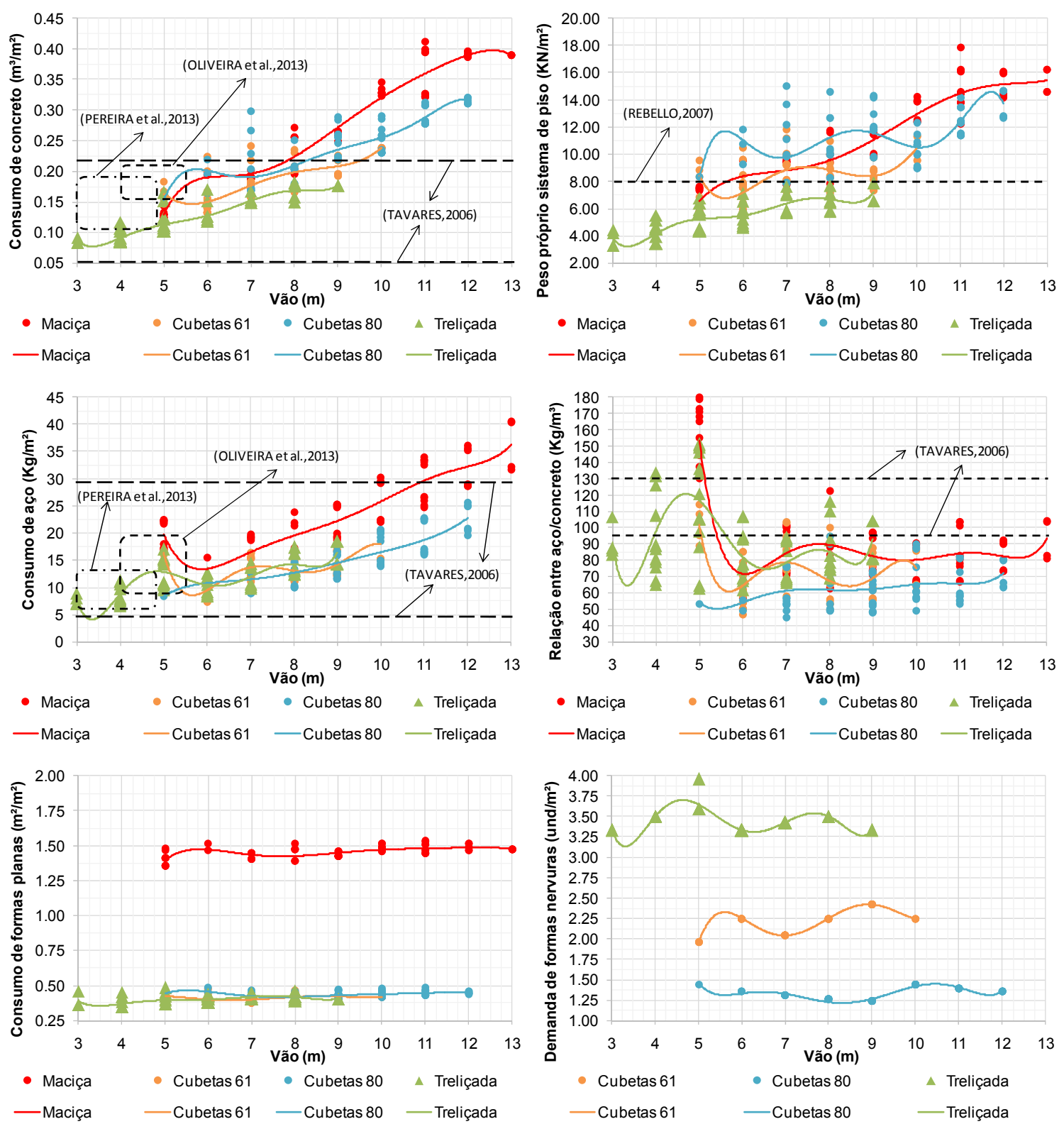

Fonte: elaborada pelos autores.

Nas expressões de [14] a [17] estão as Taxas de armadura por metro quadrado, ajustadas a partir dos dados gerados, referente às tipologias de piso com laje bidirecional. Estas expressões lineares demonstraram um desvio máximo em relação os dados geradores da ordem de $\pm 25 \%$.

$$
\begin{aligned}
& \text { TAmac }=0,32 \cdot f_{c k}-3,87 \cdot L_{v}+1,67 \cdot q_{s c}-0,52 \cdot d+0,35 \cdot b_{v}+0,57 \cdot h_{v}-4,67 \\
& \text { TAcub61 }=0,006 \cdot f_{c k}+2,08 \cdot L_{v}+1,15 \cdot q_{s c}-0,05 \cdot d-0,01 \cdot b_{v}-0,02 \cdot h_{v}-4,84 \\
& \text { TAcub80 }=0,03 \cdot f_{c k}+1,24 \cdot L_{v}+1,41 \cdot q_{s c}-0,09 \cdot d+0,12 \cdot b_{v}+0,10 \cdot h_{v}-10,80 \\
& \text { TAtre40 }=0,03 \cdot f_{c k}+1,72 \cdot L_{v}+0,87 \cdot q_{s c}-0,07 \cdot d+0,27 \cdot b_{v}-0,07 \cdot h_{v}-4,66
\end{aligned}
$$


Figura 5 - Índices de desempenho dos sistemas de piso com laje unidirecional.
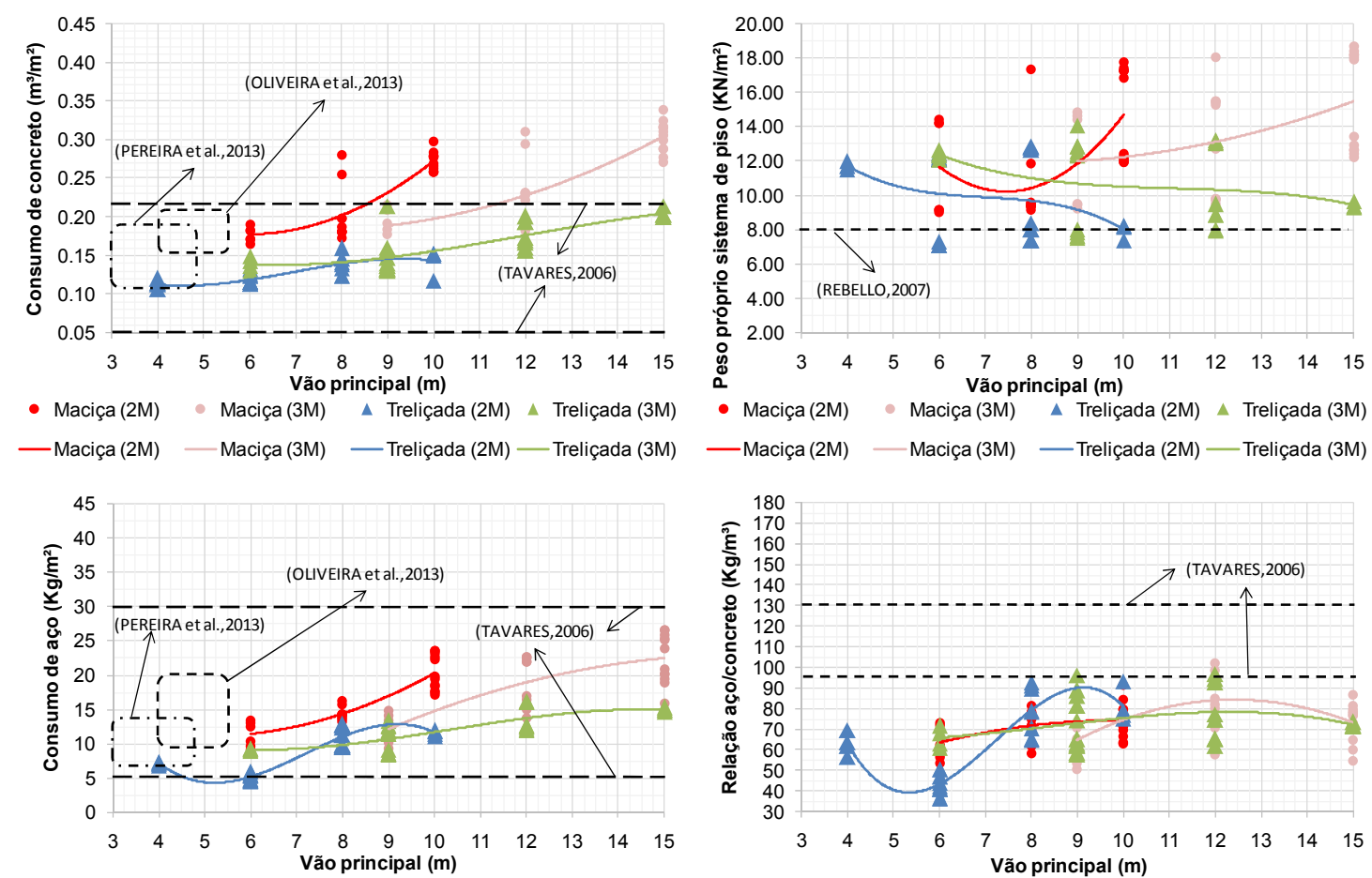

- Maciça (2M) - Maciça(3M) \ Treliçada (2M) \ Treliçada (3M)

- Maciça(2M) - Maciça(3M) \ Treliçada (2M) \& Treliçada (3M) — Maciça (2M) — Maciça (3M) —Treliçada (2M) — Treliçada (3M)

— Maciça (2M) — Maciça (3M) —Treliçada (2M) — Treliçada (3M)
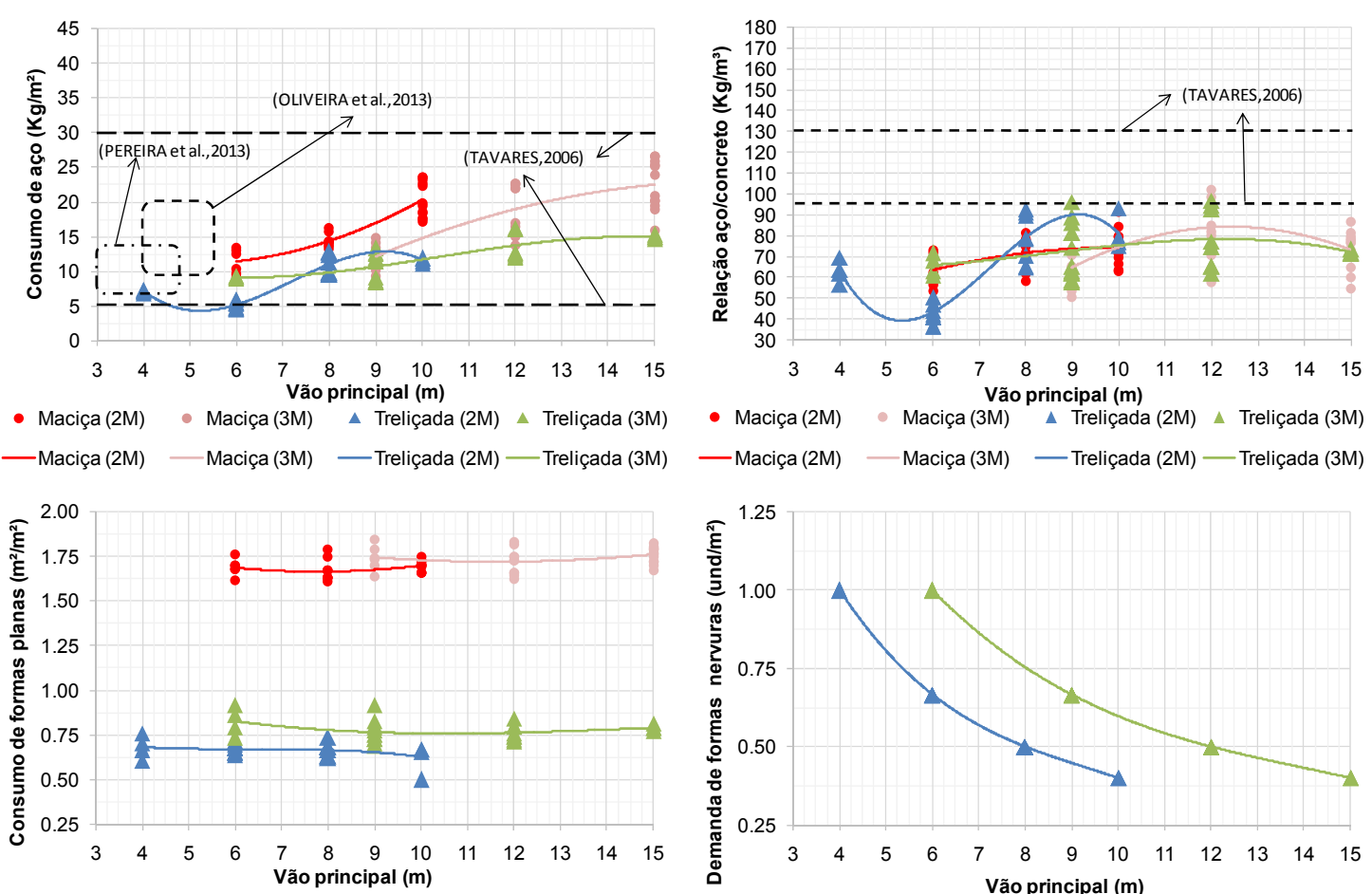

— Maciça (2M) — Maciça (3M) — Treliçada (2M) — Treliçada (3M)

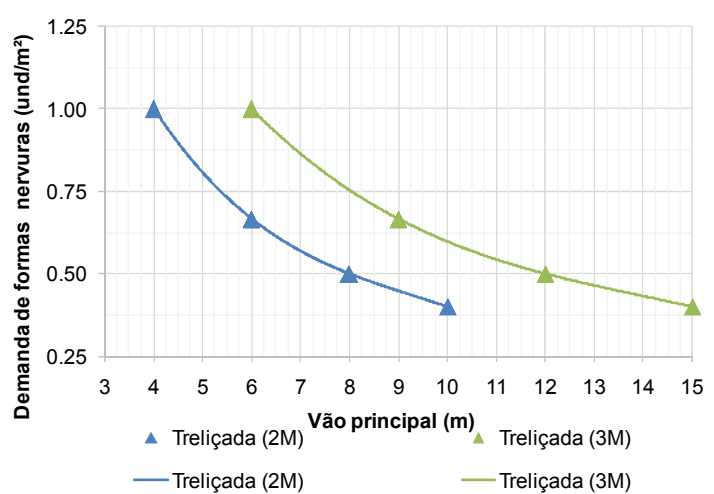

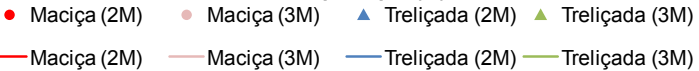

Fonte: elaborada pelos autores.

As expressões de [18] a [21] fornecem as taxas de aço obtidas pelo ajuste linear dos dados processados referentes aos sistemas de piso com laje unidirecional maciça ou treliçada. Estas funções ajustadas apresentaram um desvio máximo em relação aos dados da ordem de $\pm 20 \%$.

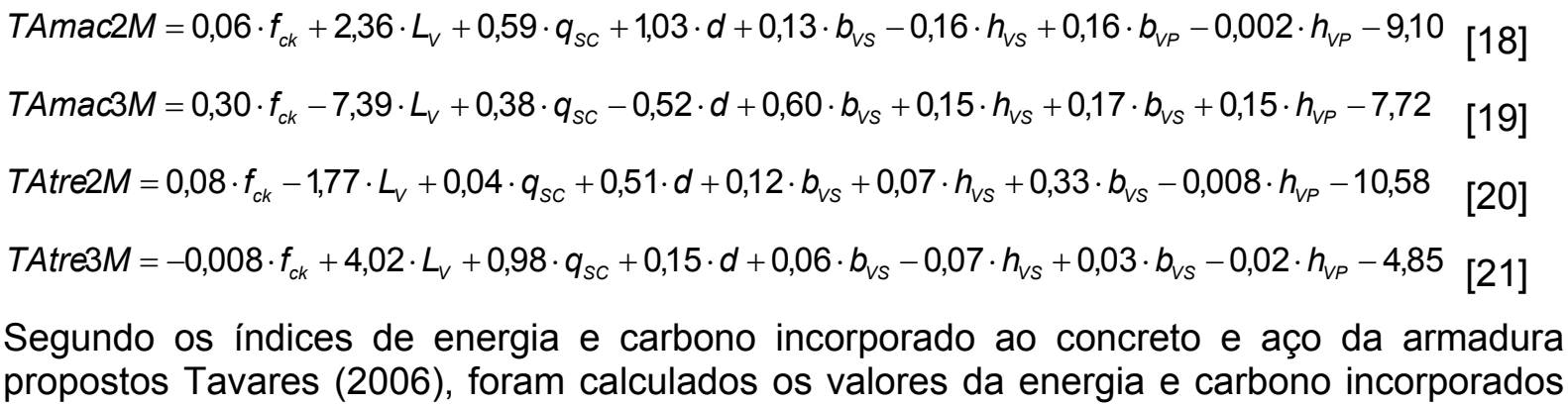


nos protótipos gerados, identificando as taxas por área de planta. Estes resultados estão ilustrados pelas figuras 6 e $7 \mathrm{com}$ contornos representativos a valores de referência relatados por outros autores.

Figura 6 - Energia e CO2 incorporado nos sistemas de piso com laje bidirecional.
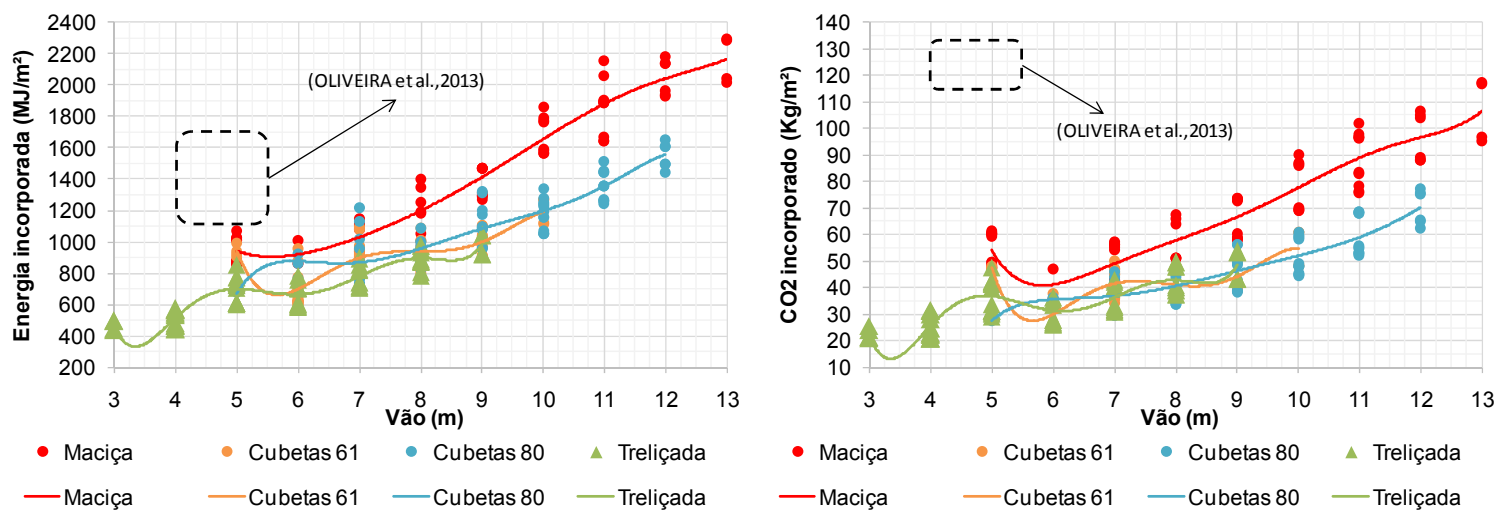

Fonte: elaborada pelos autores.

Figura 7 - Energia e CO2 incorporado nos sistemas de piso com laje unidirecional.
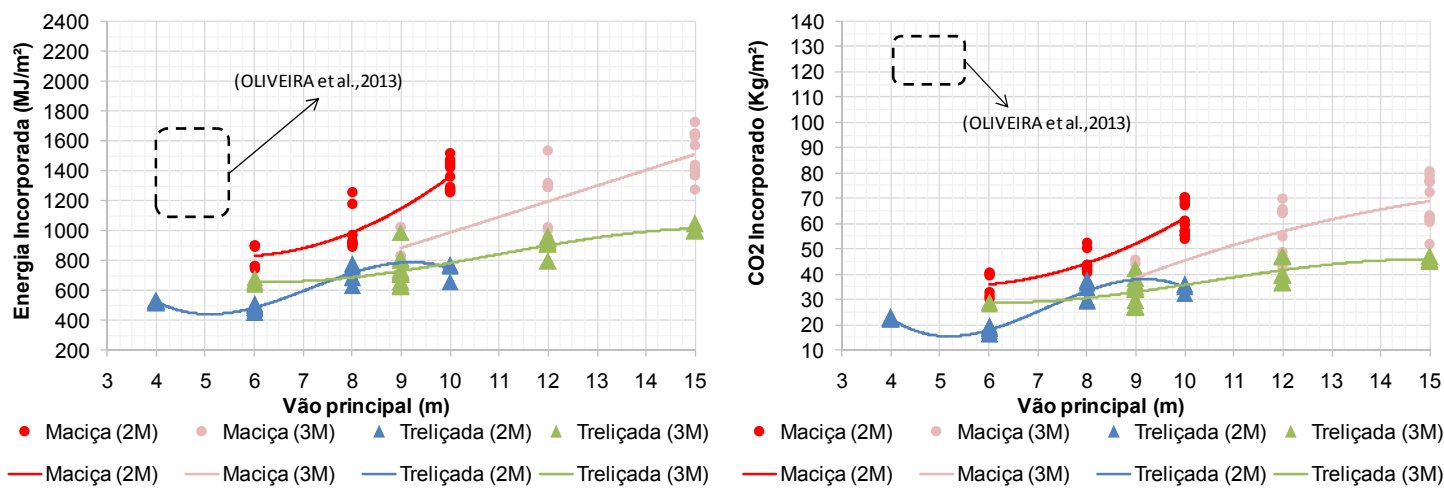

Fonte: elaborada pelos autores.

As regiões marcadas nos gráficos das figuras 4 a 7 localizam os níveis de consumo de materiais como também dos níveis de energia e $\mathrm{CO}_{2}$ incorporados nas estruturas multipavimentos completas, isto é, contendo o sistema de piso e o sistema vertical de pilares. Por isto, há a possibilidade de que a observação das diferenças destes níveis relatados pela literatura em relação aos resultados obtidos neste estudo seja, dentre outros fatores, devido à ausência da contabilidade dos índices de consumo de material, energia e $\mathrm{CO}_{2}$ incorporados dos pilares e/ou sistemas verticais neste estudo.

\section{CONCLUSÕES}

Os ábacos e índices de consumos apresentados tentam antecipar para o profissional projetista as reais consequências que cada decisão tomada na fase de concepção pode acarretar, buscando projetos mais conscientes e concisos. Nos gráficos que ilustram as taxas de consumo de material dos sistemas de piso estudados, observa-se valores inferiores de consumo em relação às taxas relatadas na literatura. Vale salientar que as taxas relatadas pela literatura se referem a todo o prédio, isto é, com a inclusão dos pilares. Em estudos futuros deve ser verificado as taxas de pilares para obtenção de dados para comparação estes e outros valores de referencia da literatura, com isso poderá ser 
verificado se as diferenças observadas neste estudo entre as taxas encontradas são devidas apenas aos pilares, se ocorrem devido à modulação da estrutura ou a outros fatores.

Percebe-se que o peso próprio dos diversos sistemas de piso estudados variam bastante de valor de acordo com a tipologia e sobretudo de acordo com o vão. Verifica-se que as lajes maciças são dotadas de maior peso próprio, demonstrando assim ser um sistema menos eficiente. Pôde ser verificado através deste estudo que o menor consumo de material está atrelado ao uso de lajes nervuradas, sobretudo as treliçadas. Contudo, não é possível dizer que os sistemas de piso com lajes nervuradas são os mais econômicos em todos os casos.

No que tange ao TIC, a conexão do banco de dados de índices de desempenho, com um software da plataforma BIM, pode modernizar o processo de projetação, principalmente dos profissionais de arquitetura. Onde no momento da construção do modelo virtual, o projetista terá consciência das consequências de cada decisão, definindo os melhores parâmetros para a edificação com base em seu contexto de implantação e sua futura utilização.

\section{AGRADECIMENTOS}

Os autores agradecem à FAPESB pela bolsa de iniciação científica concedida. Os autores também agradecem à TQS pelo apoio à esta pesquisa.

\section{REFERÊNCIAS}

ASSOCIAÇÃO BRASILEIRA DE NORMAS TÉCNICAS. NBR 6118: Projeto de Estruturas de concreto - Procedimento. Rio de Janeiro, 2003.

1980.

NBR 6120: Cargas para o cálculo de estruturas de edificações. Rio de Janeiro,

ALLEN, E.; IANO, J. The architect's studio companion: rules of thumb for preliminary design. 3rd Ed., John Wileyand Sons, 2002.

CARVALHO, R. C.; PINHEIRO, L. M. Cálculo e detalhamento de estruturas usuais de concreto armado. Volume 2. Ed. PINI, 2.ed, 2013.

CHING, F. D. K.; ONOUYE, B. S.; ZUBERBUHLER, D. Sistemas Estruturais Ilustrados: padrões sistemas e projeto.Bookman, 2010.

CORKILL, P. A. Preliminary Structural Design Charts for Architects. Architectural Science Review, 12:1, 15-20, 1969.

ENGEL, H. Sistemas Estruturais. Ed. Gustavo Gili, 2001.

GRAF, H. F.; TAVARES, S. F. Energia incorporada dos materiais de uma edificação padrão brasileira residencial.In: I' CONGRESSO DE INOVAÇÃO, TECNOLOGIA E SUSTENTABILIDADE, 2010. Brusque-SC, 2010. p. 7.

ITEC - INSTITUTO DE TECNOLOGÍA DE LA CONSTRUCCÍON. Base de dados BEDEC. Disponível em:< http://itec.es/nouBedec.e/bedec.aspx>Acessado em: 19 maio 2015.

MACDONALD, A. J. Structuraldesign for architecture. Architectural Press, 1998. 
OLIVEIRA, F. R. M.; SILVA, M. G.; GOMES, V. Indicadores de desempenho funcional, ambiental e econômico de sistemas de lajes em concreto. $55^{\circ}$ Congresso Brasileiro do Concreto, 2013.

PEREIRA, R. L.; VINHAIS, G. C.; ARAÚJO, Y. R. O. Análise de indicadores de projetos estruturais de edifícios de múltiplos pavimentos. $55^{\circ}$ Congresso Brasileiro do Concreto, 2013.

REBELLO, Y. C. P.Bases para projeto esturural na arquitetura. Ed. Zigurate, 2. ed, 2007.

SILVA, F. T. Estudo sobre sistemas de piso com lajes bidirecionais em concreto armado. $55^{\circ}$ Congresso Brasileiro do Concreto, 2013.

TAVARES, S. F. Metodologia de análise do ciclo de vida energético de edificações residenciais brasileiras. Tese de doutorado do programa de pós-graduação em engenharia civil UFSC, 2006 